\title{
Effect of hydraulic coefficient on membrane performance for rejection of emerging contaminants
}

\author{
V.S. Babu ${ }^{\mathrm{a}}$, Mahesh Padaki ${ }^{\mathrm{a}, \mathrm{b}, *}$, Laveena P. D'Souza ${ }^{\mathrm{a}}$, Sébastien Déon ${ }^{\mathrm{c}}$, R. Geetha Balakrishna ${ }^{\mathrm{a}, *}$, \\ A.F. Ismail ${ }^{\mathrm{d}}$ \\ ${ }^{\text {a }}$ Centre for Nano and Material Sciences, Jain University, Jain Global Campus, Bangalore 562112, India \\ b National University of Science and Technology "MISIS", Moscow 119049, Russia \\ ${ }^{c}$ Institut UTINAM (UMR CNRS 6213), Université de Bourgogne Franche-Comté, 16 route de Gray, 25030 Besançon Cedex, France \\ d Advanced Membrane Technology Research Center (AMTEC), Universiti Teknologi Malaysia, 81310 Skudai, Johor Bahru, Johor, Malaysia
}

The efficient removal of Endocrine Disruptive Chemicals (EDCs) namely oxybenzone and atrazine using poly-sulfone (PSf) composite membranes is reported in this article. A negatively charged hydrophilic mixed matrix membrane was prepared by using Polyaniline modified halloysite nanotubes (PANi-HNT) and polysulfone. The X-ray diffraction (XRD) and attenuated total reflectance infrared (ATR-IR) technique confirm the conversion of Halloysite nanotubes to Polyaniline modified halloysite nanotubes and their presence in membrane matrix. The microvoids observable in the SEM images depict the internal structure of the membrane. Further, increasing in the water uptake and decreasing in the contact angle with respect to increasing the concentration of Polyaniline modified halloysite nanotubes confirm the enhancement of the membrane hydrophilicity. This hydrophilicity increases for higher concentrations of PANi-HNT leads to a significant improvement of the water flux. The removal efficiency of prepared mixed matrix membranes was found to be $98 \%$ for oxybenzone and $50 \%$ for atrazine. The separation process was discussed in terms of adsorption coefficient, membrane charge and mean pore size. Considering the performances highlighted in this study, the proposed membranes appear usable for the removal of EDCs
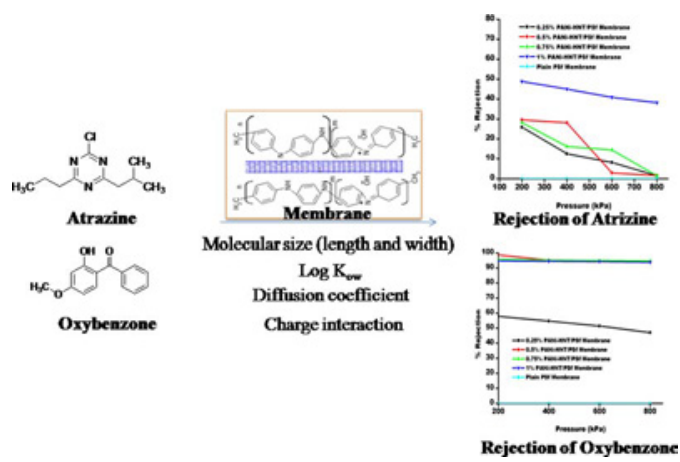

\footnotetext{
* Corresponding authors at: Centre for Nano and Material Sciences, Jain University, Jain Global Campus, Bangalore 562112, India (M. Padaki).

E-mail addresses: sp.mahesh@jainuniversity.ac.in (M. Padaki), br.geetha@jainuniversity.ac.in (R. Geetha Balakrishna).
} 


\section{Introduction}

The chemicals or natural by-products in the environment that mimic the hormones in the body are called Endocrine Disruptive Chemicals (EDCs) [1]. They have major impacts on human and wild life due to their influence on the networks of endocrine glands such as pituitary, thyroid, parathyroid, adrenals, and pancreas [2]. Human activity can sometimes introduce many endocrine compounds into the environment, which are capable of disturbing the endocrine system of aquatic, wildlife and sometimes humans. EDCs can also cause imbalance in immune system resulting in reproductive disorders. Moreover, certain types of cancer, birth defects and neurological disorders can also be induced by these contaminants. The foremost sources of EDCs are fish, meat, industrial waste water, dairy products, cosmetics and cleaning products, pesticides, herbicides and insecticides. These man-made compounds or natural products act on human body by fitting into hormone receptors, blocking the normal hormone or acting instead of the normal hormone in an irregular manner $[3,4]$.

Oxybenzone and atrazine are the major EDCs present in the environment. Oxybenzone is used as a broad-band UV filter in many concentrations ranging up to $10 \%$ in sunscreen products. It is usually used either alone or in combination with other UV filters .since it absorbs UVB (290-320 nm) and short-wave UVA (320-400 nm). It is also incorporated into environment by many other types of cosmetic products at concentrations ranging between 0.05 and $0.5 \%$ when used for photoprotection [5]. When it is exposed to UV light, it was found to undergo oxidation and form an intermediate semiquinone which inactivates the anti-oxidant enzyme thioredoxin reductase, resulting in homeostasis of the epidermis [6]. On the other hand, atrazine is an EDC that alters the natural hormone system in animals [7]. It is widely used in agriculture as an herbicide. Although it is the most widely used herbicide in US [8], its use remains controversial because of drinking water contamination. Atrazine also causes cancer, reproductive disorder, neurotoxicity, menstrual problems and birth defects. The half-life of the chemical was found to be in the range of 13 to 261 days in surface soil, and therefore, it can slowly migrate to underground water [9].

Considering their toxicity, there is a lot of researches devoted to the development of new methodologies or processes for the elimination of hazardous organic moieties. Among them, membrane separation is getting thoughtful attraction from the scientific community, perhaps due to the simple synthetic procedure and high separation efficiency [10-13]. PANi-HNT is a widely studied polymer for its electrical and optical properties, and it has been extensively used in many applications such as sensors, rechargeable batteries, anticorrosion coatings and selective membranes [14,15]. Their inclusion in PSf membranes allows a modification of their properties leading to a significant improvement of performances such as water flux and separation of contaminants [16]. These membranes can be recycled many times, which is ecofriendly and cost effective [17].

Present work involves the synthesis of various Wt.\% PANi-HNT/PSf mixed matrix membranes (MMM) by phase inversion process. To the best of our knowledge, the rejection of oxybenzone and atrazine by PANi-HNT/PSf membranes was rarely investigated in the literature. Moreover, the manuscript provides a deep discussion about the parameters involved in the separation of emerging contaminants. XRD and ATR-IR spectroscopy were used to study the microstructure of the membranes and the morphology was also characterized by SEM images. Hydrophilicity of the various membranes was investigated by contact angle measurement and their performances in EDCs rejection were explored using different $\mathrm{Wt} \% \mathrm{PANi}-\mathrm{HNT} / \mathrm{PSf}$ membranes. Finally, the rejection measurements were also extended to the filtration of solutions containing EDCs mixed with water collected from a lake.

\section{Experimental section}

\subsection{Materials}

The various compounds used in this study are 2-Hydroxy-4methoxy-benzophenone (oxybenzone) (Merck), 2-chloro-4-(ethylamino)-6-(isopropylamino)-s-triazine (atrazine) (Merck), polysulfone (Mw: 32,000 Da) (Sigma-Aldrich), 1-methyl-2-pyrrolidone (NMP) (Merck), HNT (Sigma-Aldrich), ammonium persulfate and HCl (Merck). All the chemicals used were of the reagent grade, and the solvents were dried and distilled before use as per the standard protocols.

\subsection{Methods}

\subsubsection{Preparation of PANi-HNT}

Polyaniline coated HNT was synthesized using in situ polymerization technique following the method reported by Zhang et al.(2008) with slight modifications [18]. $1.5 \mathrm{~g}$ of HNT was sonicated in $100 \mathrm{~mL}$ of distilled water to which $3 \mathrm{~mL}$ of aniline and $270 \mathrm{~mL}$ of $1 \mathrm{M} \mathrm{HCl}$ were added with continuous stirring at $10^{\circ} \mathrm{C}$. Temperature plays a critical role in this reaction as it avoids sudden dissociation of the acid. A solution of ammonium persulfate $(7.5 \mathrm{~g}$ in $300 \mathrm{~mL}$ of $1 \mathrm{M} \mathrm{HCl})$ was slowly added to the reaction mixture over a period of $90 \mathrm{~min}$, and the mixture was then kept for $4 \mathrm{~h}$ at room temperature without any disturbance. Then, dark colored PANi modified HNT with chlorine salt was filtered and washed with distilled water. Finally the product was stirred with aqueous ammonium hydroxide to remove any excess of acid and to neutralize the product. In this process, chlorine is replaced by $\mathrm{OH}$ to form free base [19]. The obtained PANi-HNT was dried in oven at $70{ }^{\circ} \mathrm{C}$ for $48 \mathrm{~h}$. Scheme 1 depicts the chemical reactivity of prepared PANiHNT.

\subsubsection{Preparation of PANi-HNT/PSf composite membranes}

The PANi-HNT/PSf composite membranes with desired weight percent of PANi-HNT were prepared by phase inversion process [20]. The desired amount of PANi-HNT was taken into 16 mLof N-methylpyrollidine (NMP) of $99 \%$ purity, sonicated for $5 \mathrm{~h}$ at room temperature and stirred about30 min for uniform dispersion. Polysulfone (PSf) (MW $=32,000 \mathrm{Da})$ was discharged into the solution mixture and stirred for $24 \mathrm{~h}$ to obtain a viscous solution, which was casted on glass plate using glass rod and dipped in a coagulation bath to get composite membrane sheet of thickness ca.1 to $1.2 \mu \mathrm{m}$. The thickness of the membranes was kept uniform using side tape. Finally, membranes were washed several times with distilled water to remove excess of solvent that might be present during the preparation process and further by dipping into distilled water for $24 \mathrm{~h}$ [21]. The different ratios of polysulfone, PANi-HNT and NMP used for the preparation of the different membranes are showcased in Table 1.

\subsection{Characterization of the PANi-HNT and mixed matrix membranes}

\subsubsection{ATR-IR and XRD}

Attenuated total reflectance-infrared (ATR-IR) spectra were recorded on a Perkin Elmer FTIR 1650 Spectrophotometer in the range 400 and $4000 \mathrm{~cm}^{-1}$. The PANi-HNT and mixed matrix membranes were compared by ATR-IR; the membranes were thoroughly dried for $24 \mathrm{~h}$ in a vacuum desiccator before recording the IR spectra. X-ray diffraction patterns of the PANi-HNT and mixed matrix membranes were recorded using a Shimadzu XRD-model 7000 with Cuka radiation scan rate of 1 $\min ^{-1}$, and the crystalline nature of HNT on mixed matrix membranes was studied by XRD technique.

\subsubsection{SEM analysis}

SEM images of the membrane cross section were used to study the membrane morphology. The cross section was observed using a Scanning Electron Microscope (Technai 10 Phillips). The samples were 


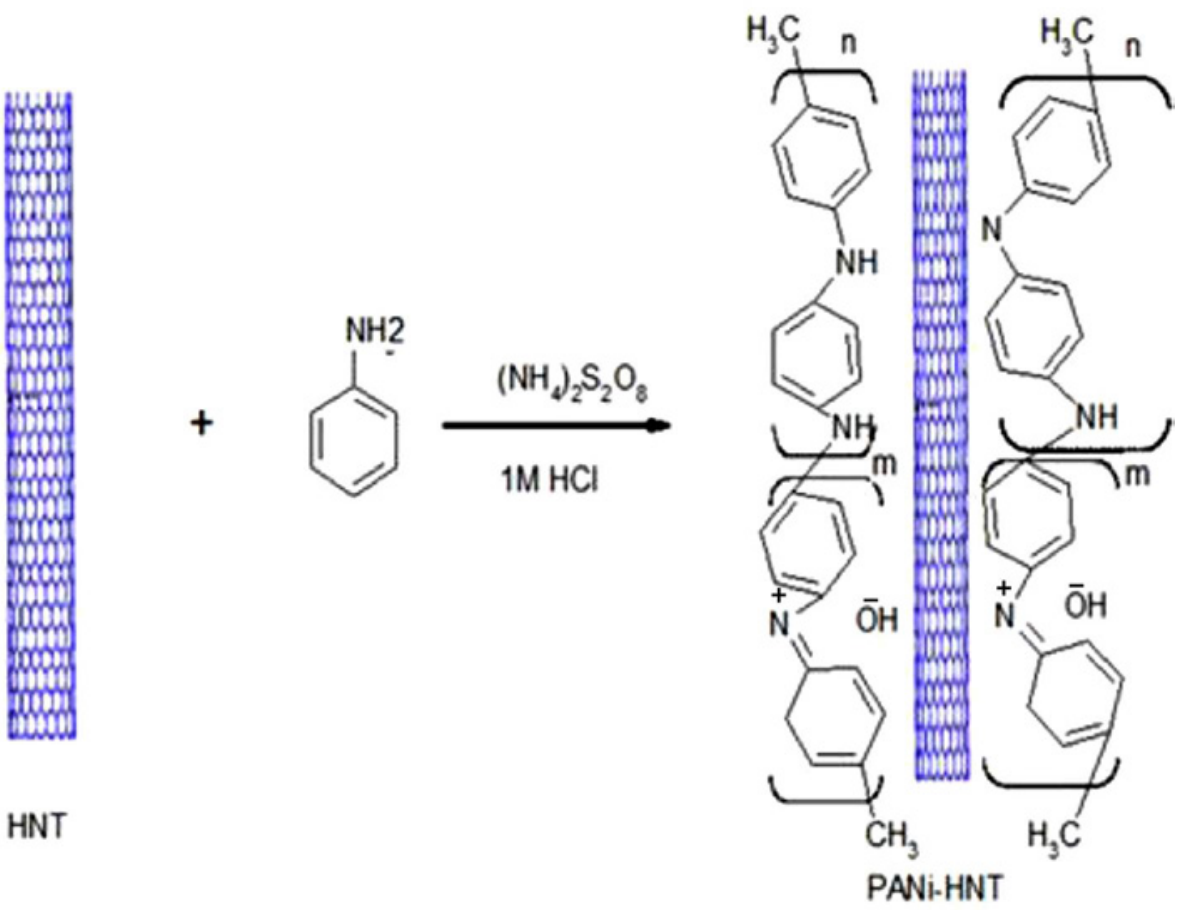

Scheme 1. Preparation of PANi-HNT by chemical reaction

Table 1

Different concentration of PSf and PANi-HNT in the various membranes.

\begin{tabular}{llll}
\hline Membrane type & Polysulfone & PANi-HNT & NMP \\
\hline $0.25 \%$ PANi-HNT/PSf membrane & $18.75 \%$ & $0.25 \%$ & $81 \%$ \\
$0.5 \%$ PANi-HNT/PSf membrane & $18.5 \%$ & $0.5 \%$ & $81 \%$ \\
$0.75 \%$ PANi-HNT/PSf membrane & $18.25 \%$ & $0.75 \%$ & $81 \%$ \\
$1 \%$ PANi-HNT/PSf membrane & $18 \%$ & $1 \%$ & $81 \%$ \\
\hline
\end{tabular}

prepared by freezing and fracturing the membranes with liquid nitrogen to provide smooth break of the membrane cross section. The prepared samples were affixed on carbon tape, making an aluminum disc stand and then sputter-coated with $15 \mathrm{~nm}$ of gold prior to SEM analysis.

\subsubsection{Wettability study of membranes}

The water uptake and contact angle are the two main two parameters to determine the hydrophilicity of the membranes [22]. The water uptake of the prepared membrane was performed by recording the change in the weight of the dry membrane and the membrane which was soaked in distilled water. Membranes were thoroughly rinsed with distilled water, and then dried in vacuum desiccators with a vacuum of $120 \mathrm{~mm} \mathrm{Hg}$ for $24 \mathrm{~h}$. The dried membrane was sliced into small pieces of $c a .1 \mathrm{~cm}^{2}$ area and was further immersed in distilled water. Eventually, these pieces of membranes were taken out after $24 \mathrm{~h}$ interval of time, and excess water on the surface was gently removed by a blotter. Finally, the weight of the soaked membranes was quickly measured. And the water uptake percentage was calculated using the Eq. (1).

$\%$ water uptake $=\left(\frac{\mathrm{W}_{\mathrm{w}}-\mathrm{W}_{\mathrm{d}}}{\mathrm{W}_{\mathrm{d}}}\right) \times 100$

where, $\mathrm{W}_{\mathrm{w}}$ and $\mathrm{W}_{\mathrm{d}}$ are the weights of wet and dried membranes, respectively.

The contact angle between the water and the membrane was measured by using a FTA-200 Dynamic contact angle measurement by sessile drop method. A water droplet was placed on the membrane at five different places and the average value is reported.

\subsubsection{Ion exchange capacity (IEC)}

The determination of the ion exchange capacity consists in measuring the number of counter ions exchangeable in membrane in milliequivalents of $\mathrm{H}^{+}$per weight of the dry membrane. The ion exchange capacity gives information about the density of ionizable functional groups present on the membrane. These ionizable groups are majorly responsible for the charged nature of the membrane and for membrane conductivity [23]. The membranes were cut into $1 \mathrm{~cm}^{2}$ square shaped pieces, dried well and weight was noted. Then, they were kept in a $10 \mathrm{~mL}$ of $1 \mathrm{M} \mathrm{HCl}$ solution for $24 \mathrm{~h}$ for exchange of cations and rinsed with distilled water. Finally, the membranes were kept in $10 \mathrm{~mL}$ of $1 \mathrm{M}$ $\mathrm{NaCl}$ solution for $24 \mathrm{~h}$. The ion exchange capacity was calculated by obtained $\mathrm{HCl}$ solution through titration method as described in the literature [24]. The IEC was calculated using Eq. (2)

$\mathrm{IEC}=\left(\frac{\mathrm{AB}}{\mathrm{m}_{\mathrm{dry}}}\right) \times 100$

where, $\mathrm{A}=$ Concentration of the $\mathrm{NaOH}$ used, $\mathrm{B}=$ Volume of $\mathrm{NaOH}$ solutionconsumed during the titration and $\mathrm{M}_{\mathrm{dry}}=$ weight of the dry membrane.

\subsection{Membrane performance study}

\subsubsection{Rejection of oxybenzone and atrazine}

The rejections of EDCs were carried out by using self-constructed dead-end filtration unit. The experimental filtration procedure proposed by Jyothi et al. [25] was followed for rejection measurements. Prepared membranes of desired Wt.\% were cut into round shape of $5 \mathrm{~cm}^{2}$ and fixed at the bottom of the unit. The unit is then filled with $500 \mathrm{~mL}$ of solution containing $3 \mathrm{ppm}$ of afore-mentioned EDCs. The filtration experiments were run separately for oxybenzone and atrazine. For each applied pressure, $20 \mathrm{~mL}$ of the solution was collected for determination of EDCs concentration. Solution were analyzed at $\lambda_{\max }=235 \mathrm{~nm}$ for oxybenzone and at $\lambda_{\max }=225 \mathrm{~nm}$ for atrazine, using a UV-Visible Spectrophotometer. The applied pressure was varied from 200 to $1000 \mathrm{kPa}$ with the difference of $200 \mathrm{kPa}$. For each pressure, a new membrane and feed solution was used. The percentage of rejection was calculated using Eq. (3). 
$\%$ rejection $=\left(1-\frac{C_{p}}{C_{f}}\right) \times 100$

where, $C_{p}$ and $C_{f}$ are the permeate and feed concentrations, respectively.

The above procedure was repeated by using each of the following membranes one by one, viz, $0.25 \mathrm{wt} \%$ of PANi-HNT/PSf membrane, $0.5 \mathrm{wt} \%$ of PANi-HNT/PSf membrane, $0.75 \mathrm{wt} \%$ of PANi-HNT/PSf membrane, and $1 \mathrm{wt} \%$ of PANi-HNT/PSf membrane for oxybenzone and atrazine.

\subsubsection{Pore size identification}

In this paper, the mean pore radius $r_{p}$ of the various membranes was estimated by fitting rejection of atrazine and oxybenzone with a hydrodynamic model that considers only steric exclusion. This approach has been implemented many times in literature and the value of pore radius is usually adjusted in Eq. (4) to fit a complete rejection curve of a neutral solute such as glucose [26].

$\%$ rejection $=1-\frac{\varphi K_{c}}{1-\left[\left(1-\varphi K_{c}\right)(\exp (-P e))\right]}$

$P e$ denotes the Peclet permeation number defined as:

$P e=\frac{K_{c} \Delta P}{8 K_{d} D_{\infty} \eta} r_{p}^{2}$

With $K_{c}$ and $K_{d}$ the hindrance factors for convection and diffusion, respectively and $\phi$ the steric partition coefficient defined by [27]:

$K_{d}=1-2.30 \lambda+1.154 \lambda^{2}+0.224 \lambda^{3}$

$K_{c}=(2-\varphi)\left(1+0.054 \lambda-0.988 \lambda^{2}-0.441 \lambda^{3}\right)$

$\varphi=\left(1-\frac{r_{s}}{r_{p}}\right)^{2}$

In this study, the pore radius has been identified for each pressure separately to discuss the exclusive phenomena governing rejection of atrazine and oxybenzone.

\subsubsection{Rejection of oxybenzone and atrazine from a lake water}

The rejections of EDCs were also carried out by using feed solution collected from a nearby lake. The filtration procedure is identical to that previously described. The feed solution was prepared in the laboratory by mixing the compounds in the lake water. Water was collected from the lake and stored in laboratory for $24 \mathrm{~h}$ without disturbing. Afterwards, upper layer of water was collected and used for the preparation of feed sample. The rejection of EDCs was studied in the same conditions that those used for synthetic solutions.

\section{Results and discussion}

\subsection{Characterization of the membranes}

Fig. 1 depicts the XRD spectra of HNT, PANi-HNT and composite membranes. The XRD spectra of HNT with a set of characteristic peaks at $13^{\circ}, 21^{\circ}$ and $27^{\circ}$ of two theta, whereas XRD spectrum of PANi-HNT, which shows peaks with decreased intensity compared with HNT spectra. Further, the peaks were shifted towards left to $12^{\circ}, 20^{\circ}$ and $27^{\circ}$ of two theta, which may be attributed to the fact that the interaction of in situ generated polymer chain into the intra molecular layer of HNT [28]. The XRD spectrum of HNT and PANi-HNT matches with literature standard, which clearly shows the successful modification of HNT to PANi-HNT [18].The XRD spectrum of PSf membrane showing broad peaks at $18^{\circ}$ of two theta, while the XRD spectra of $1 \%$ PANi-HNT/PSf membrane. In the case of these mixed matrix membranes, PANi-HNT peaks were not observed due to low concentration in the polymer matrix.

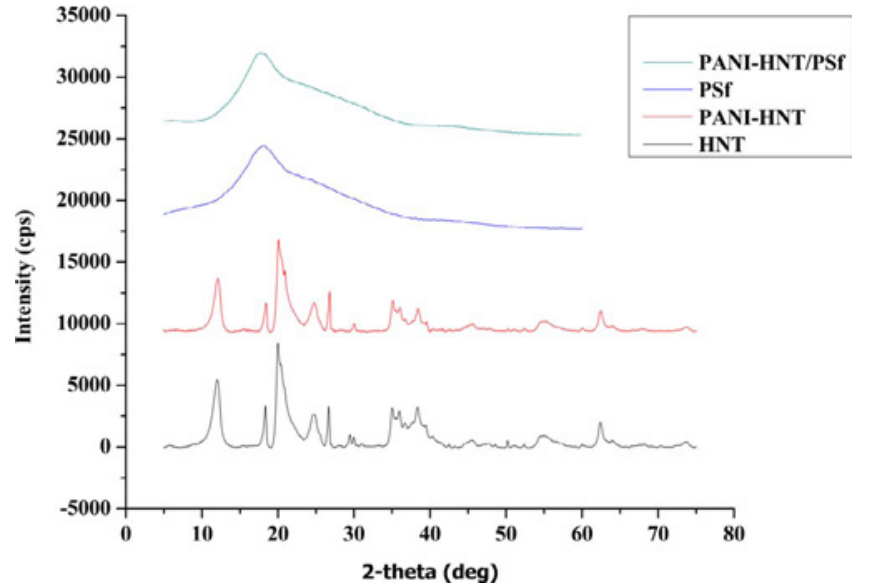

Fig. 1. XRD spectra of HNT, PANI-HNT, PSf membrane and 0.5\% PANi-HNT/PSf membrane.
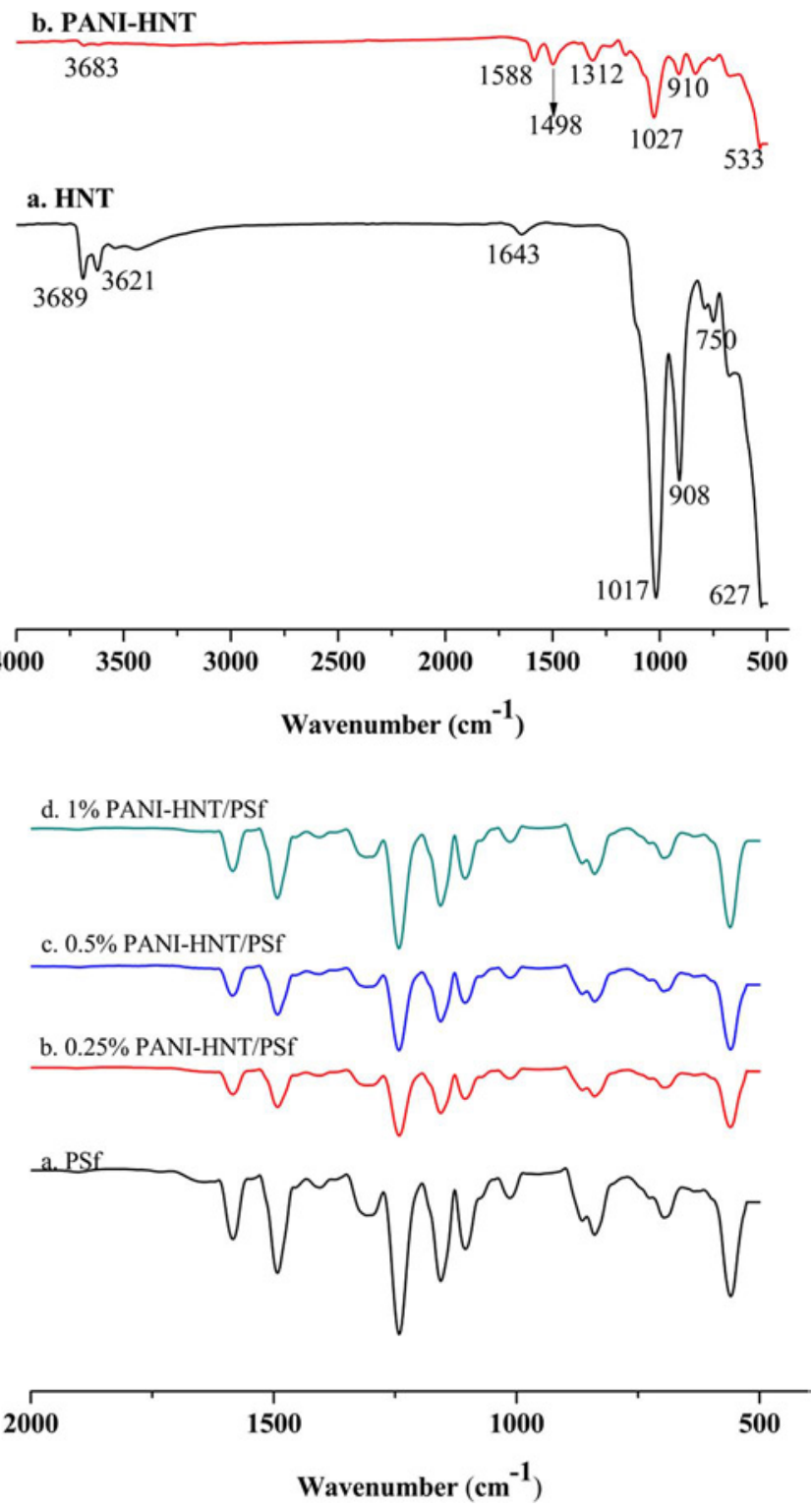

Fig. 2. ATR-IR spectra of (a) HNT and PANi-HNT (b) membranes of plane PSF and PANiHNT/PSf. 

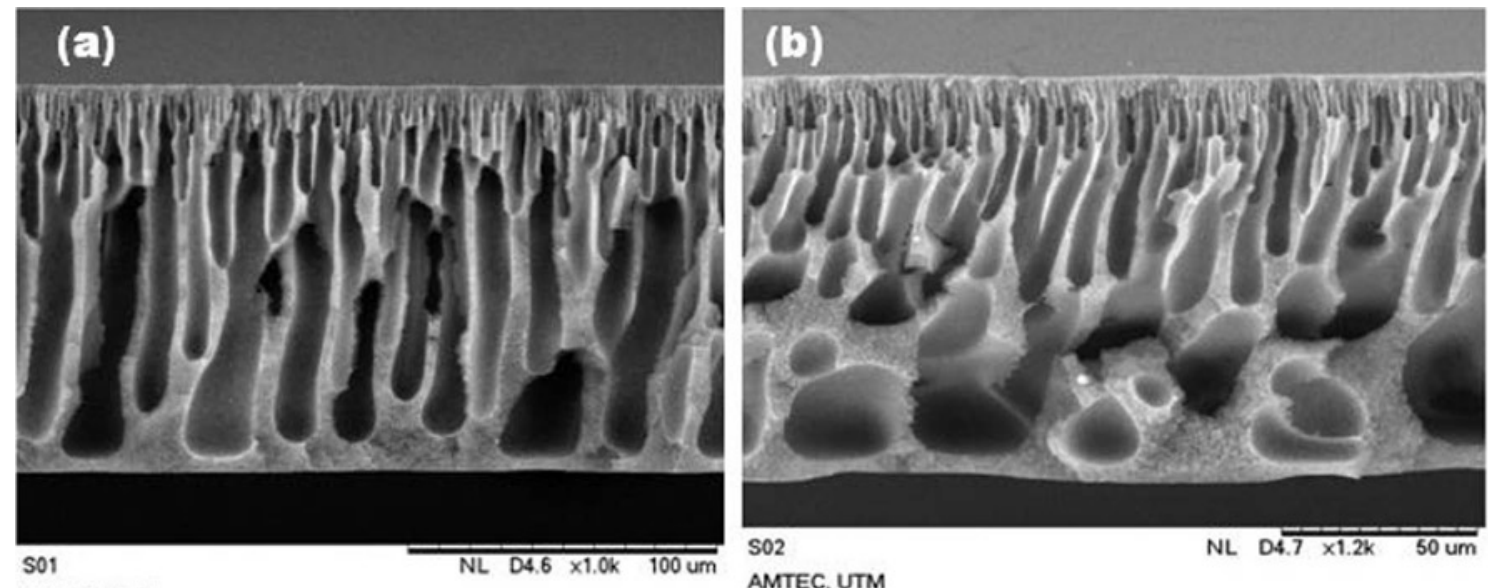

AMTEC, UTM AMTEC, UTM

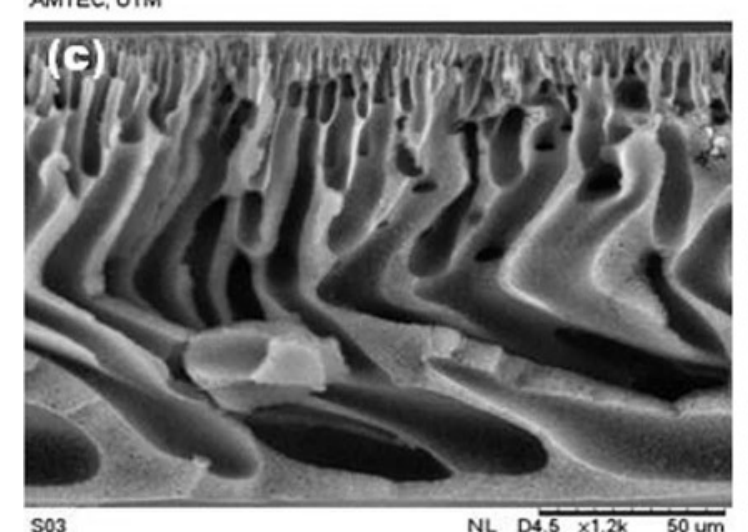

$\mathrm{S} 03$

AMTEC, UTM

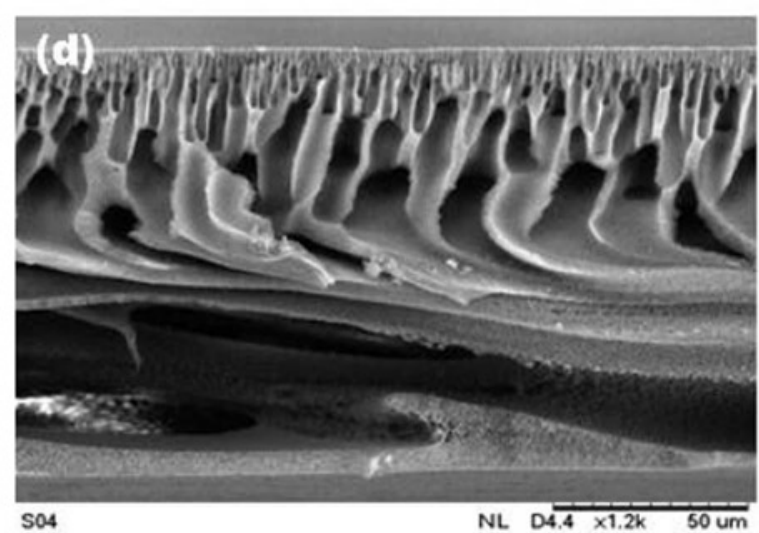

AMTEC, UTM

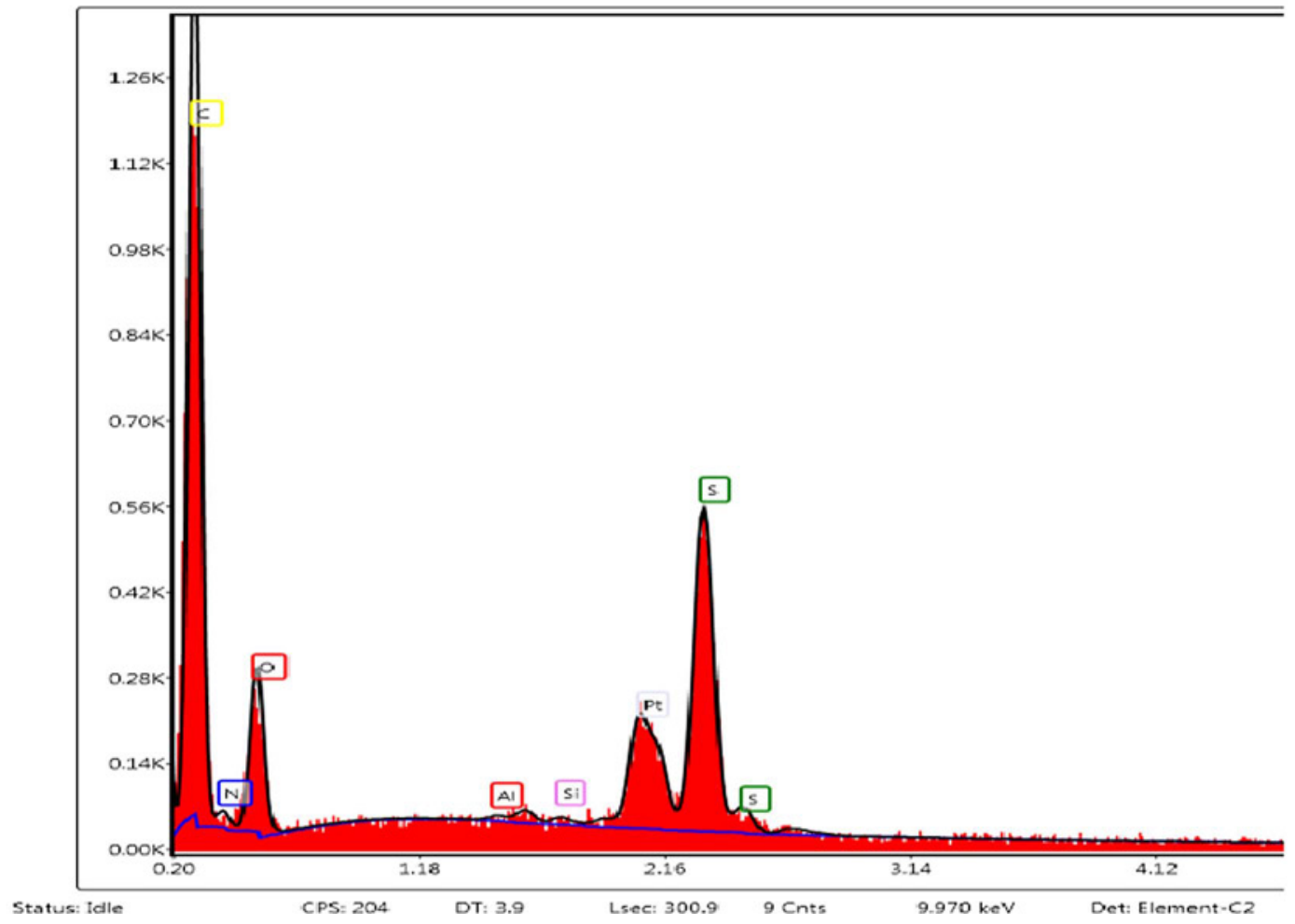

Fig. 3. SEM monographs of (a) Plain PSf cross section (b) 0.25\% PANi-HNT/PSf cross section (c) 0.5\% PANi-HNT/PSf cross section (d) $1 \%$ PANi-HNT/PSf cross section (e) EDAX analysis of the membrane 
Fig. 2 portrays the stacked ATR-IR spectra of PSf membrane, PANiHNT and mixed matrix membranes. Of particular interest, Fig. 2a exhibits a band characteristic for NH stretching vibrations of PANi-HNT in the range $3600-3683 \mathrm{~cm}^{-1}$. Two distinguishing bands observed at 1588 and $1498 \mathrm{~cm}^{-1}$ were attributed to $\mathrm{C}=\mathrm{C}$ stretching of benzenoid ring and $\mathrm{C}=\mathrm{N}$ stretching vibrations of quinoid rings, respectively. Further, the peaks at $1312 \mathrm{~cm}^{-1}$ correspond to $\mathrm{C}-\mathrm{N}$ stretching modes. These all peaks are not observed in HNT spectrum, which confirms the formation of PANI HNT. Fig. 2b represents the IR spectra of membranes which shows bending vibrations of $\mathrm{C}-\mathrm{H}$ stretch at $1156 \mathrm{~cm}^{-1}$ and stretching vibrations in the range $1105 \mathrm{~cm}^{-1}$ due to $\mathrm{O}=\mathrm{S}=\mathrm{O}$ of the PSF. IR spectra of $0.5 \mathrm{wt} \%$ PANi-HNT/PSf and $1 \mathrm{wt} \%$ PANi-HNT/PSf membranes. The presence of PANi-HNT on PSf membrane could modify the resulting bands either by change in the relative intensity or by shifting some of the bands this is evident as $\mathrm{C}=\mathrm{N}$ and $\mathrm{C}=\mathrm{C}$ bands have been shifted from 1589 and $1508 \mathrm{~cm}^{-1}$ to 1585 and $1503 \mathrm{~cm}^{-1}$.This observation can be attributed to the intra molecular bonding between PANi-HNT and PSf.

Figure 3 illustrates the cross-section images of PSf and mixed matrix membranes. EDAX study of the membrane was performed and it shows presence of $\mathrm{Al}, \mathrm{Si}$, and $\mathrm{N}$ in the membrane, which confirms the presence of PANi-HNT in the membrane matrix. Of particular interest, Fig. 3(b-d) exhibit mixed matrix membranes with the concentration of PANi-HNT increases from 0.25 to $1 \%$, respectively. The cross-section reveals the presence of a dense layer, a finger like middle layer and a bottom supportive layer system. The finger like projections are narrow in PSf membrane, whereas increasing the concentration of PANi-HNT, this projection became broad while micro channels on the surface layer become thinner. In the bottom layer, however, the macrovoids are longer with the increase in the concentration of the PANi-HNT, below the finger like projections. There are two important parameters, effectively for such macrovoids during phase inversion are; i) hydrophilicity and ii) viscosity of the casting solution [28]. Hydrophilicity increases the intake of non-solvents (water) in the solvent/non-solvents exchange and higher the viscosity decreases the rate of solvent exchange which causes macrovoids [29]. In this case the PANi-HNT is hydrophilic in nature meanwhile metal oxides decrease the viscosity of the casting solution. Hence, macrovoids are larger with respect to higher concentration of PANi-HNT The hydrophilicity of the membranes also supports the above phenomenon, and was confirmed by water uptake and contact angle studies. In higher concentration, the water movement is irregular because of more hydrophilic interaction doing phase inversion. Hence, twisted finger like projections were observed in higher concentration of PANi-HNT membranes.

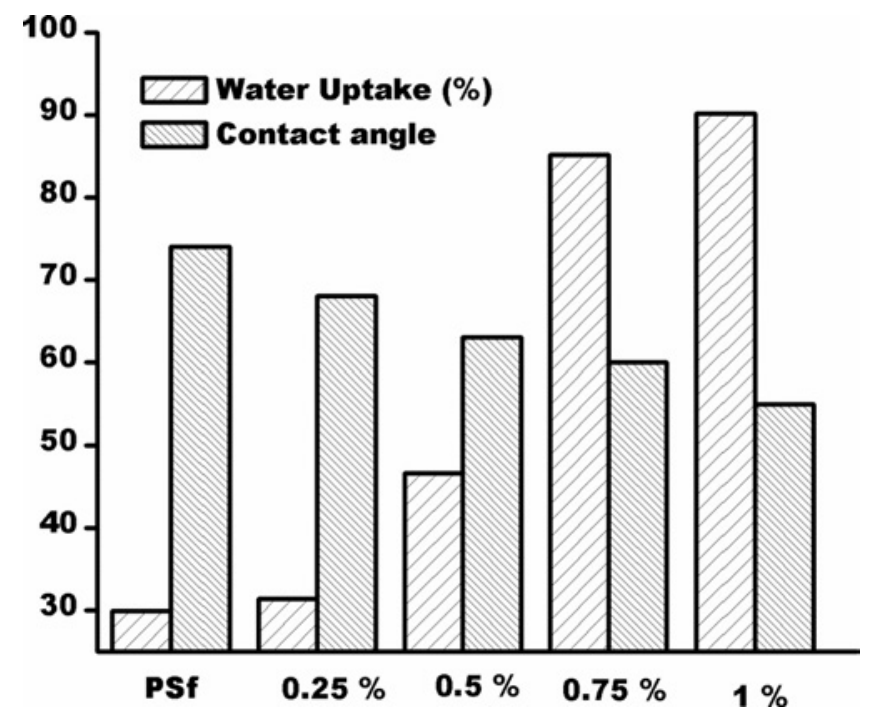

Fig. 4. Water uptake and contact angle of the membranes
Fig. 4 demonstrates the water uptake and contact angle for the prepared membranes of various concentrations of PANi-HNT distributed over PSf membranes. The contact angle decreases from $74 \pm 2$ to $55 \pm 4^{\circ}$ as the concentration of the PANi-HNT increases from 0 to $1 \%$. The water uptake was increased from 29.9 to $31.43 \%$, after the incorporation of $0.25 \%$ PANi-HNT over PSf membrane. Similarly, the water uptake increases substantially upon the increase in the concentration of PANi-HNT, that is, 0.5\% PANi-HNT, 0.75\% PANi-HNT, and $1 \%$ PANi-HNT to $46.67 \%, 85.15 \%$ and $90.18 \%$, respectively. The presence of PANi-HNT on the surface of the PSf membrane drastically improves the water uptake/hydrophilicity. Hydroxyl polarity is able to interact with water molecules through Van der Waal's force and hydrogen bonding, which helps to enhance the hydrophilicity of the membrane [30]. In PANi-HNT, the presence of $\mathrm{NH}_{2}$ groups leads to the increase of Van der Waal's interactions with water molecules. However, it is also possible that the higher affinity of PANi-HNT for water compared to polysulfone, which increases the penetration velocity of water into mixed matrix membrane during the phase inversion process [28]. Hence, mixed matrix membranes are hydrophilic in nature as compare to plane PSf membrane.

PANi-HNT is a charged molecule, and therefore, IEC of the membrane (given in Table 2) is also an important characteristic to enhance the membrane performance. The amine group from PANi has capability to do the ion exchange. Meanwhile, prepared PANi-HNT is in basic form with $\mathrm{OH}^{-}$counter ions. Hence, prepared membranes exhibit the charge interaction phenomenon in performance. Table 2 shows the ion exchange capacity of the membranes. IEC capacity depends on the concentration of PANi-HNT; higher concentration of the PANi-HNT enhances the exchange capacity.

\subsection{Water flux study}

Pure water flux study is one of the important parameters in judging the porous nature of the membrane [31]. Fig. 5 shows the pure water flux at different operating pressures for various PANi-HNT/PSf membranes. In the case of the plain PSf membrane and $0.25 \%$ PANi-HNT/ PSf membrane, no water flux is observed even at $800 \mathrm{kPa}$ of pressure indicating the hydrophobic nature of these membranes, which hinders the penetration of water. However, a high water flux was observed in the cases of higher percentage of PANi-HNT membranes with the increase in pressure. These results are almost linear with respect to transmembrane pressure. It was observed that there is a constant increase in the water flux with respect to increase in the weight percentage of PANi-HNT. This is perhaps due to the hydrophilic nature of the substance. Hydrophilicity increases with respect to concentration of PANi-HNT and the same trend was followed in the water flux study. The highest concentration i.e. 1\% PANi-HNT evinced maximum water flux of $40.15 \mathrm{~L} / \mathrm{m}^{2} \mathrm{~h}$ at $200 \mathrm{kPa}$ and $86.5 \mathrm{~L} / \mathrm{m}^{2} \mathrm{~h}$ at $800 \mathrm{kPa}$ pressure. The water flux was found to decrease with the decrease in concentration of PANi-HNT irrespective of the pressure considered. Hence, the productivity of the membrane depends basically on its hydrophilic character.

\subsection{Rejection study using PANi-HNT/PSfmembrane}

The rejection of trace organic molecules by membranes depends on

Table 2

Ion exchange capacity of the mixed membrane.

\begin{tabular}{ll}
\hline Membrane type & IEC (m equ/g) \\
\hline $0.25 \%$ PANi-HNT/PSf membrane & 13.88 \\
$0.5 \%$ PANi-HNT/PSf membrane & 15 \\
$0.75 \%$ PANi-HNT/PSf membrane & 15.6 \\
1\% PANi-HNT/PSf membrane & 15.6 \\
\hline
\end{tabular}




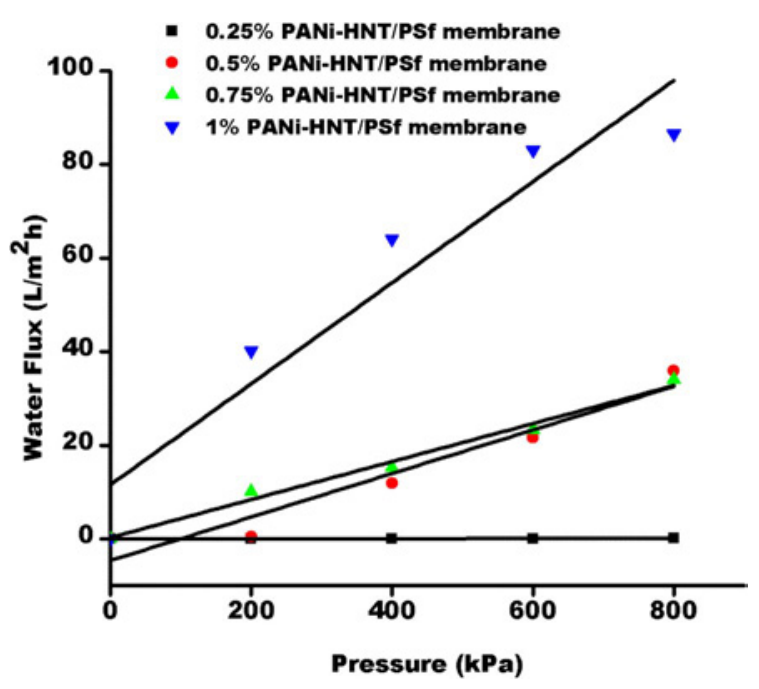

Fig. 5. Pure water flux of the membranes

the feed parameters like molecular weight (MW), molecular size (length and width), acid dissociation constant (pKa), hydrophobicity/hydrophilicity $\left(\log \mathrm{K}_{\mathrm{ow}}\right.$ ), diffusion constant (Dp) and membrane properties such as pore size (MWCO value), surface charge (IEX), or hydrophobicity/hydrophilicity (measured as contact angle and water uptake) and surface morphology [32]. PANi-HNT is more hydrophilic and negatively charged. Hence, PSf /PANi-HNT composite membrane were used for the removal of atrazine and oxybenzone.

Fig. 6 shows the extent of rejection of oxybenzone with respect to various applied pressures. The results reveal that the membrane of lower concentration of $0.25 \%$ PANi-HNT/PSf evinced a rejection of $c a$. $55 \%$ of these organics. The $0.5,0.75$ and $1 \%$ PANi-HNT/PSf membranes evidenced $95 \%$ of rejection as higher hydrophilicity enhances the rejection. The membrane performance was studied for various pressures, from 200 to $800 \mathrm{kPa}$, however, the same rejection values were found for all the applied pressures.

The extent of rejection of atrazine with respect to applied pressure is showcased in Fig. 7. The 1\% PANi-HNT/PSf membrane displayed maximum rejection of $c a .50 \%$ was observed at a lower pressure of $200 \mathrm{kPa}$ while the same performance was continuously found even at higher pressure. In the cases of $0.25,0.5$ and $0.75 \%$ PANi-HNT/PSf membranes, a rejection of around $30 \%$ was observed at lower pressure of $200 \mathrm{kPa}$. The rejection increases with increase in $\log \mathrm{K}_{\mathrm{ow}}$ for both the

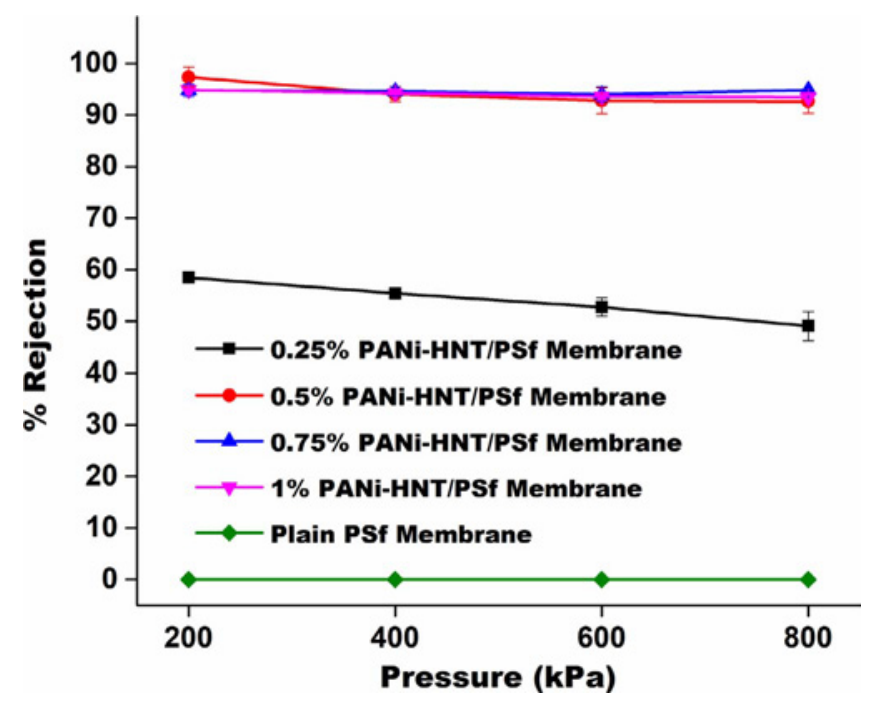

Fig. 6. Rejection of Oxybenzone at different pressure

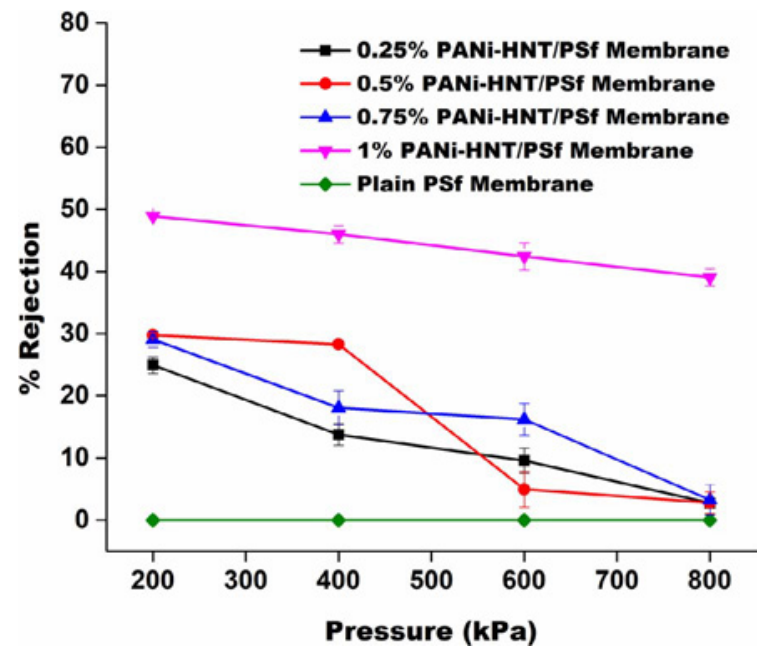

Fig. 7. Rejection of Atrazine at different pressure

compounds (oxybenzone and atrazine having Log $\mathrm{K}_{\mathrm{ow}}>2.5$ ), which indicates their hydrophobic nature. Therefore, higher rejection was observed with highly hydrophilic membranes [33]. In the instance of atrazine (Fig. 7), the 1\% PANi-HNT/PSf membrane exhibitedca. $50 \%$ of rejection; however, decreasing concentration of PANi-HNT of $0.25,0.5$ and $0.75 \%$ illustrated $30 \%$ of the atrazine rejection. Furthermore, the rate of the rejection decreases with an increase in the applied pressure. The anomalous behavior of the separation results was justified using properties of organic compounds.

Almost $95 \%$ and $35 \%$ of rejections were observed in the cases of oxybenzone and atrazine, respectively, using the same membranes. The transport mechanism in the membrane separation was affected by the properties of feed solution [32]. Table 3shows the physical properties of the oxybenzone and atrazine. The pKa value of atrazine is 1.7 which is acidic in nature, and it can undergo chemical reaction with the membranes as it is a chloro substituted derivative. Hence, it can damage the surface of the membrane by increasing the pore size. Surface which cause enlarging the pore size, meanwhile, diffusion coefficient of the atrazine is higher than the oxybenzone. Moreover, oxybenzone is considerably larger in size than atrazine and the steric exclusion is also strongly lower with the latter. Hence, the rejection of atrazine is drastically lower than that of oxybenzone.

Each PANI-HNT/Psf membranes shows significantly higher rejection than the Psf membranes and it is especially true when the percentage of PANI-HNT is high (1\%). Moreover, rejection obtained with the $1 \%$ PANI-HNT/Psf is found to be notably higher than those reported in literature for UF membranes $(0-10 \%$ for atrazine [34,35] and $70 \%$ for oxybenzone [35]) and even for loose NF membranes (30-40\% for

Table 3

Physical properties of atrazine and oxybenzone.

\begin{tabular}{|c|c|c|}
\hline Properties & Atrazine & Oxybenzone \\
\hline Molecular Weight & $215.68 \mathrm{Da}$ & $228.24 \mathrm{Da}$ \\
\hline $\begin{array}{l}\text { Molecular size } \\
\text { (length and } \\
\text { width) }\end{array}$ & $0.788 \mathrm{~nm}$ & $3.16 \mathrm{~nm}$ \\
\hline $\begin{array}{l}\text { Acid dissociation } \\
\text { constant (pKa) }\end{array}$ & 1.7 & 7.6 \\
\hline $\log \mathrm{K}_{\mathrm{ow}}$ & 2.7 & 3.79 \\
\hline $\begin{array}{l}\text { Diffusion } \\
\text { coefficient }\end{array}$ & $5.4 \times 10^{-10} \mathrm{~m}^{2} / \mathrm{s}$ & $3 \times 10^{-8} \mathrm{~cm}^{2} / \mathrm{s}$ \\
\hline Structure & & $\mathrm{OH}$ \\
\hline
\end{tabular}




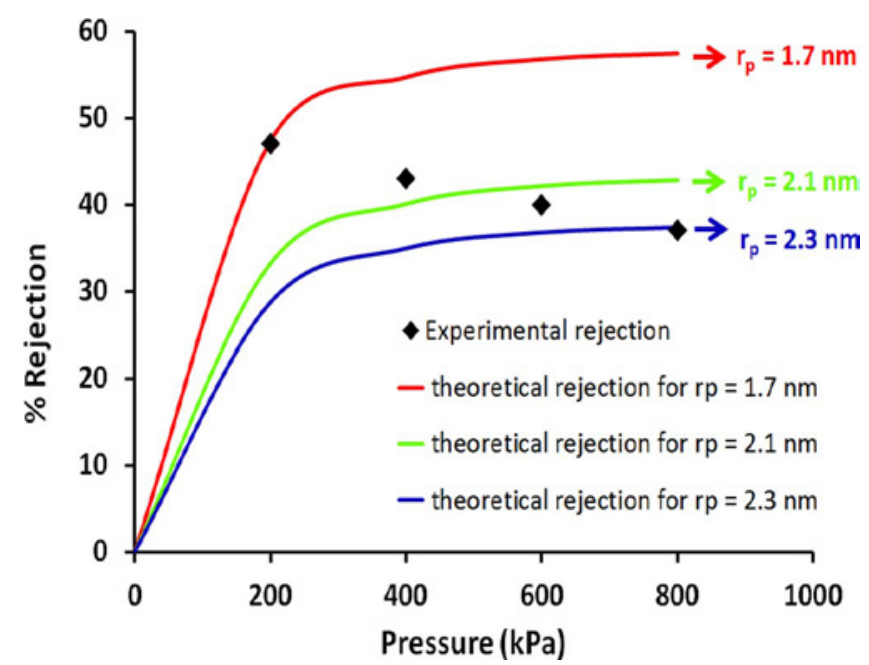

Fig. 8. Estimation of the mean pore radius from experimental rejection of atrazine by the $1 \%$ PANI-HNT/PSf membrane

atrazine $[34,35]$ and $70 \%-80 \%$ for oxybenzone [36]). For this reason, EDCs are usually removed from contaminated solution by hybrid membrane processes, such as membrane bioreactor and/or membrane distillation [37]. However, considering the performances highlighted in this study, PANI-HNT-Psf membranes seems to be a relevant alternative to hybrid processes due to their modified properties.

The rejections obtained for the various filtration experiments were used to estimate the mean pore radius of the two membranes by considering only steric exclusion (Eqs. 45.2). It is expected that only one pore radius can describe a whole curve of rejection, but the rejection decrease when pressure increases does not allow the determination of a unique pore size. Thus, pore radius was estimated for three pressures corresponding to 200, 500 and $800 \mathrm{kPa}$, as it is depicted in Fig. 8 in the case of atrazine rejection by the $1 \%$ PANI-HNT/PSf membrane. The values estimated for all conditions are summarized in Table 4 for discussion.

As it can be seen in Table 4, the identified pore radius increases with pressure, which is obvious since experimental rejection was found to decrease when pressure increases. Nonetheless, identified values are close to $2-4 \mathrm{~nm}$, meaning that membranes can be classified as ultrafiltration membranes with low molecular weight cut-off. The decrease of rejection for increasing pressure being probably induced by a strong concentration polarization, it is probably more coherent to discuss the pore radii obtained at $200 \mathrm{kPa}$. From values identified with the two solutes, it can be seen that membrane exhibits different pore sizes, which is physically incoherent. This observation tends to show that rejection is probably governed by other phenomena than steric exclusion, such as electrostatic interactions. Consequently, a hydrodynamic model is not sufficient to describe rejection of such molecules and a more complex model including electric and dielectric exclusions [38] should be developed for this purpose.

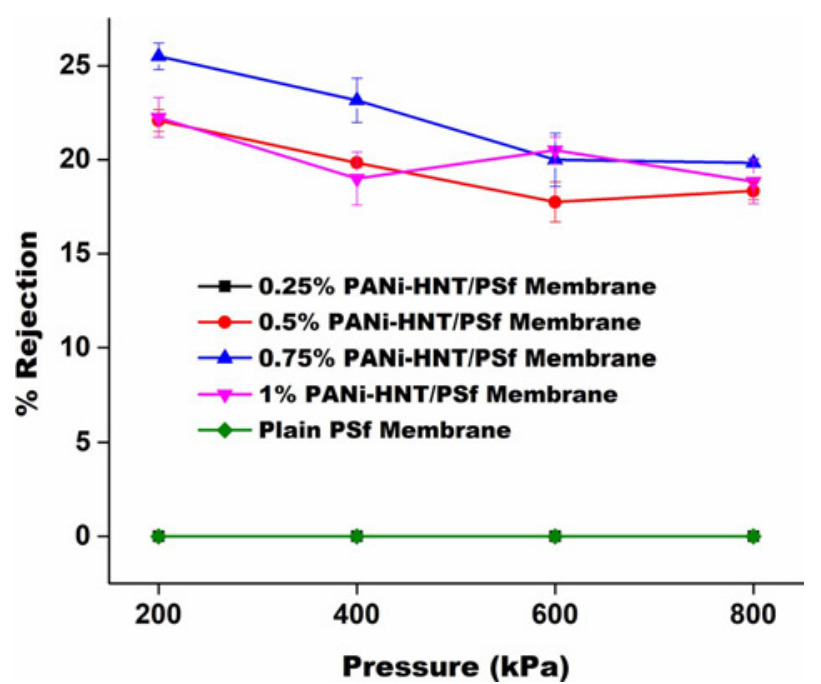

Fig. 9. Rejection of laboratory prepared field water with oxybenzone at different pressure.

\subsection{Rejection study for the laboratory prepared feed sample}

Further, the membrane was used to remove EDCs from laboratory feed samples prepared with a real water. Indeed, it was previously reported that rejection of EDCs can also vary depending on feed water chemistry [39].

The solution containing $3 \mathrm{ppm}$ of oxybenzone and atrazine were prepared using waste water collected from a lake and the rejection was investigated using the same filtration unit and procedure. Fig. 9 shows the evolution of oxybenzone rejection with respect to various applied pressure. In case of $0.25 \%$ PANi-HNT/PSf membrane, there was no rejection, but in the cases of $0.5,0.75$ and $1 \% \mathrm{PANi}-\mathrm{HNT} / \mathrm{PSf}$ an average rejection of $20 \%$ was observed. Almost the same average rejection was observed for atrazine in Fig. 10with the same mixed matrix membranes. With these samples, the rejection of atrazine and oxybenzone appears no longer to be governed by steric exclusion, leading to similar rejections although their sizes are significantly different.

With an increase in the feed water concentration, a concentration differential is created across the membrane and resulting in ahigh diffusion potential. Diffusion across the membrane is one of the main driving forces for the maximum permeation of organic compounds [32]. The recovery of the HAAS, DBP and total THM by NF membranes was studied by Chellam and Taylor, who found that the rejection decreases by increasing recovery [40]. Hence, in this case, the feed solution was full of other organic compounds. It increases the diffusion potential across the membrane which causes the falls in rejection.

\section{Conclusion}

The prepared membranes have the ability to remove the hydrophobic organic trace compounds. The polysulfone is a hydrophobic membrane and it was converted hydrophilic by mixing with PANI-HNT. The hydrophilic and negatively charged membrane showed better performances with both charge and hydrophilicity, which are the main

Table 4

Pore radius of the various membranes estimated for 3 pressures from atrazine and oxybenzone rejection.

\begin{tabular}{|c|c|c|c|c|c|c|c|c|c|c|c|c|}
\hline \multirow{2}{*}{$\begin{array}{l}\text { Membranes } \\
\text { Pressures (kPa) }\end{array}$} & \multicolumn{3}{|c|}{$\begin{array}{l}0.25 \% \\
\text { PANi-HNT/PSf }\end{array}$} & \multicolumn{3}{|c|}{$\begin{array}{l}0.5 \% \\
\text { PANi-HNT/PSf }\end{array}$} & \multicolumn{3}{|c|}{$\begin{array}{l}0.75 \% \\
\text { PANi-HNT/PSf }\end{array}$} & \multicolumn{3}{|c|}{$\begin{array}{l}1 \% \\
\text { PANi-HNT/PSf }\end{array}$} \\
\hline & 200 & 500 & 800 & 200 & 500 & 800 & 200 & 500 & 800 & 200 & 500 & 800 \\
\hline$r_{p}(n m)$ from atrazine & 2.35 & 4.5 & 8 & 2.2 & 3.9 & 8 & 2.3 & 3.9 & 8 & 1.7 & 2.1 & 2.3 \\
\hline$r_{p}(n m)$ from oxybenzone & 2.5 & 2.6 & 2.65 & 3.3 & 3.9 & 3.9 & 3.6 & 3.8 & 3.9 & 3.7 & 3.8 & 3.8 \\
\hline
\end{tabular}




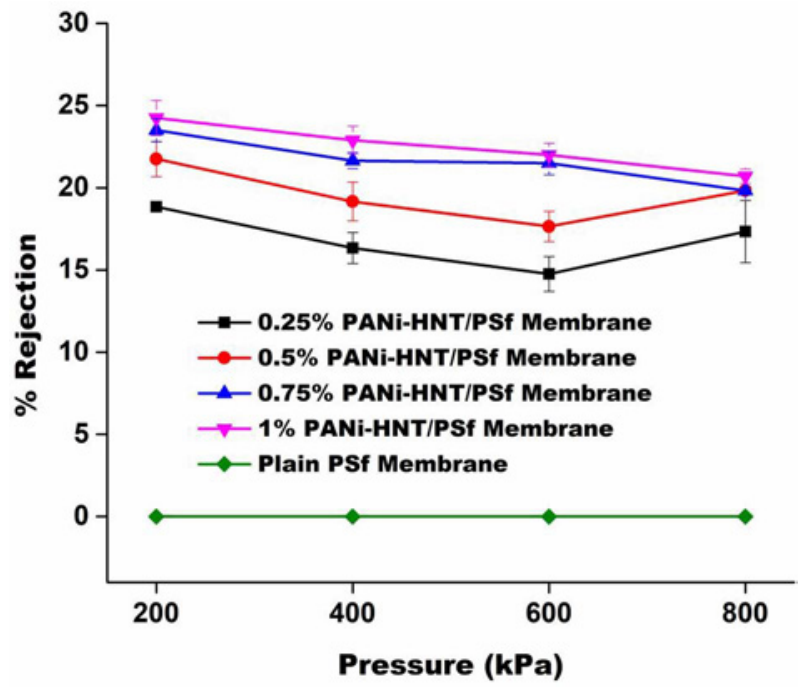

Fig. 10. Rejection of laboratory prepared field water with Atrazine at different pressure.

properties governing transport through the membrane. The water uptake and flux were higher for higher concentrations of PANi-HNT. The physical properties of the feed solution play a crucial role in the rejection studies. For instance, the $\log \mathrm{K}_{\mathrm{ow}}$ and the size of the molecule have shown a great influence on the separation performances. The compounds which are having $\log \mathrm{K}_{\mathrm{ow}}>2.8$ can be removed using these membranes. Oxybenzone showed $95 \%$ and atrizines $c a .50 \%$ of rejection by the reported membranes.

\section{Acknowledgements}

The authors sincerely acknowledge the Indian Ministry of Drinking water and Sanitation (no. 11017/33/2012-WQ), Department ofscience and technology for SERB (SB/FT/CS-045/2014), Nano mission projects (SR/NM/NS-20/2014) and the Ministry of Education and Science of the Russian Federation in the framework of Increase Competitiveness Program of NUST MISiS» (№ K3-2016-032) for financial support.

\section{References}

[1] T.M. Crisp, E.D. Clegg, R.L. Cooper, W.P. Wood, D.G. Anderson, K.P. Baetcke, J.L. Hoffmann, M.S. Morrow, D.J. Rodier, J.E. Schaeffer, L.W. Touart, M.G. Zeeman, Y.M. Patel, Environmental endocrine disruption: an effects assessment and analysis, Environ. Health. Persp. 106 (1998) 11-56.

[2] L. Sherwood, Human Physiology: From Cells to Systems, Wadsworth Pub Co, ISBN 0495391840, 1997.

[3] A.C. Johnson, J.P. Sumpter, Removal of endocrine-disrupting chemicals in activated sludge treatment works. Environmental Science and Technology, Environ. Sci. Technol 35 (2001) 4697-4703.

[4] M.B. Schlumpf, M.C. Cotton, V. Haller, B. Steinmann, Lichtensteiger, In vitro and in vivo estrogenicity of UV screens, Environ. Health. Persp. 109 (2007) 239-244.

[5] M.E. Burnett, S.Q. Wang, Current sunscreen controversies: a critical review, Photodermatol. Photoimmunol. Photomed 27 (2011) 58-67.

[6] C. Sundaram, W. Koster, K.U. Schallreuter, The effect of UV radiation and sun blockers on free radical defence in human and guinea pig epidermis, Anti. Dermatol. Res. 282 (1990) 326-331.

[7] H. Jiang, C. Adams, N. Graziano, A. Roberson, M. McGuire, D. Khiari, Occurance and removal of chloro-s-triazines in water treatment plants, Environ. Sci. Technol. 40 (2006) 3609-3616.

[8] L.P. Gianessi, M.B. Marcelli, Pesticide use in U.S. crop production 1997: "National Summary Report" National Center for Food and Agricultural Policys, 2000.

[9] A. Frank, The economics of atrazine, Int. J. Occup. Env Heal. 13 (2007) 437-445.

[10] W.S.W. Ho, K.K. Sikar, Membrane Handbook, Van Nostrand Reinhold, NY, 1992.

[11] R.W. Baker, E.L. Cussier, W. Eykamp, W.J. Koros, R.L. Riley, H. Strathmann, Membrane Separation Systems: Recent Developments and Future Directions, NDC, Park Ridge, NJ, 1991.

[12] V.T. Magalad, A.R. Supale, S.P. Maradur, G.S. Gokavi, T.M. Aminabhavi, Preyssler type heteropolyacid-incorporated highly water-selective sodium alginate-based inorganic-organic hybrid membranes for pervaporation dehydration of ethanol,
Chemic Eng. J. 159 (1-3) (2010) 75-83.

[13] V.T. Magalad, S.S. Pattanashetti, G.S. Gokavi, M.N. Nadagouda, T.M. Aminabhavi, Proton conducting properties of nanocomposite membranes of chitosan, Chem Eng. J. 189-190 (2012) 1-4.

[14] M.L. Arrascue, H.G. Maldonado, O. Horna, E. Guibal, Gold sorption on chitosan derivative, Hydrometallurgy 71 (2003) 191-200.

[15] M. Baghalha, Laeching of an oxide gold ore with chloride/hypochlorite solutions, Int. J. Miner. Process 82 (2007) 178.

[16] R.S. Murali, M. Padaki, T. Matsuura, M.S. Abdullah, A.F. Ismail, Polyaniline in situ modified halloysite nanotubes incorporated asymmetric mixed matrix membrane for gas separation, Sep. Puri. Tech. 132 (2014) 187-194.

[17] S. Tuprakay, W.J. Liengcharensit, Lifetime and regeneration of immobilized titania for photocatalytic removal of aqueous hexavalent chromium, J. Hazard. Mater. 124 (2005) 53-58.

[18] L. Zhang, T. Wang, P. Liu, Polyanilline - coated halloysite nanotubes via in-situ chemical polymerization, Appl. Surf. Sci. 255 (2008) 2091-2097.

[19] Jaroslav Stejskal, Pavel Kratochvíl, Aubrey D. Jenkins, The formation of polyaniline and the nature of its structures, Polymer 37 (1996) 367-369.

[20] M. Padaki, A.M. Isloor, P. Wanichapichart, A.M. Ismail, Preparation and characterization of sulfonatedpolysulfone and N-phthloyl chitosan blend composite cation exchange membrane for desalination, Desalination 298 (2012) 42-48.

[21] Y.H. Teow, A.L. Ahmaqd, J.K. Lim, B.S. Ooi, Preparation and characterization of PVDF/TiO2 mixed matrix membrane via in situ colloidal precipitation method, Desalination 295 (2012) 61-69.

[22] M.S. Jyothi, Vignesh Nayak, Mahesh Padaki, R. Geetha Balakrishna, Khantong Soontarapa, Eco-friendly membrane process and product development for complete elimination of chromium toxicity in wastewater, J. Hazard. Mater. 332 (2017) $112-123$.

[23] R. Vignesh Nayak, Geetha Balakrishna, Mahesh Padaki, Khantong Soontarapa Zwitterionic, Ultrafiltration membranes for As (V) rejection, Chem. Eng. J. 308 (2017) 347-358.

[24] C. Klayson, B.P. Ladewig, G.Q. Max Lu, L. Wang, Preparation characterization of sulfonatedpolyethersulfone for cation-exchange membranes, J. Membr. Sci. 368 (2001) 48-53.

[25] M.S. Jyothi, V. Nayak, M. Padaki, R.G. Balakrishna, A.F. Ismail, The effect of UV irradiation of PSf/TiO2 mixed matrix membrane for chromium rejection, Desalination 354 (2014) 189-199.

[26] A. Escoda, S. Déon, P. Fievet, Assessment of dielectric contribution in the modeling of multi-ionic transport through nanofiltration membranes, J. Membr. Sci. 378 (2011) 214-223.

[27] C.Y. Lang, P. Uchytil, R. Petrychkovych, Y.C. Lai, K. Friess, M. Sipek, M.M. Reddy, S.V. Suen, Comparison on gas separation between PES (polyethersulfone)/MMT (Na-montmorillonite) and PES/TiO 2 mixed matrix membranes, Sep. Purif. Technol. 92 (2012) 57-63.

[28] M. Padaki, D. Emadzadeh, T. Masturra, A.F. Ismail, The effect of UV irradiation on PSf/TiO2 mixed matrix membrane for chromium rejection, Desalination 362 (2015) $141-150$.

[29] V. Nayak, M.S. Jyothi, R.G. Balakrishna, M. Padaki, S. Deon, Novel modified poly vinyl chloride blend membranes for removal of heavy metals from mixed ion feed sample, J. Haz. Mat. 331 (2017) 289-299.

[30] N. Sakai, K. Fukuda, T. Shibata, Y. Ebina, K. Takada, T. Sasaki, Photoinduced hydrophilic conversion properties of titaniananosheets, J. Phys. Chem. B 110 (2006) 6198-6203.

[31] S. Fengmei, Y. Ma, J. Ma, P. Wang, W.J. Sun, Preparation and characterization of $\mathrm{PVDF} / \mathrm{TiO}_{2}$ hybrid membranes with different dosages nano $\mathrm{TiO}_{2}$, J. Memb. Sci. 389 (2012) 522-531.

[32] Y. Yoon, P. Westerhoff, S.A. Snyder, E.C. Wert, J. Yoon, Removal of endocrine disrupting compounds and pharmaceuticals by nanofiltration and ultrafiltration membranes, Desalination 202 (2007) 16-23.

[33] C. Bellona, J.E. Drewes, P. Xu, G. Amy, Factors affecting the rejection of organic solutes during NF/RO treatment -a literature review, Water Res. 38 (2004) 2795-2809.

[34] E.C. Devitt, F. Ducellier, P. Cote, M.R. Wiesner, Effects of natural organic matter and the raw water matrix on the rejection of atrazine by pressure-driven membranes, Water Res. 32 (9) (1998) 2563-2568.

[35] Y. Yoon, P. Westerhoff, S. Snyder, E. Wert, Nanofiltration and ultrafiltration of endocrine disrupting compounds, pharmaceuticals and personal care products, J. Membr. Sci. 270 (1-2) (2006) 88-100.

[36] A. Comerton, R. Andrews, D. Bagley, C. Hao, The rejection of endocrine disrupting and pharmaceutically active compounds by NF and RO membranes as a function of compound and water matrix properties, J. Membr. Sci. 313 (1-2) (2008) 323-335.

[37] K. Wijekoon, F. Hai, J. Kang, W. Price, T. Cath, L. Nghiem, Rejection and fate of trace organic compounds (TrOCs) during membrane distillation, J. Membr. Sci. 453 (2014) 636-642.

[38] S. Déon, A. Escoda, P. Fievet, P. Dutournié, P. Bourseau, How to use a multi-ionic transport model to fully predict rejection of mineral salts by nanofiltration membranes, Chem. Eng. J. 189-190 (2012) 24-31.

[39] S. Kim, K.H. Chu, Y. Al-Hamadani, C.M. Park, M. Jang, D.-H. Kim, M. Yu, J. Heo, Y. Yoon, Removal of contaminants of emerging concern by membranes in water and wastewater: a review, Chem. Eng. J. 335 (2018) 896-914.

[40] S. Chellum, J.S. Taylor, Simplified analysis of contaminant rejection during ground and surface water nanofiltration under the information collection rule, Water Res. 35 (2001) 2460. 